American Journal of Nursing Science
2020; 9 (4): $239-243$
http://www.sciencepublishinggroup.com/j/ajns
doi: 10.11648 /j.ajns.20200904.23
ISSN: 2328 -5745 (Print); ISSN: $2328-5753$ (Online)

\title{
Analysis on Related Factors and Nursing Interventions of Vasovagal Syncope During Ultrasound-guided PICC
}

\author{
Ying Wu ${ }^{1}$, Jinai $\mathrm{He}^{1}$, Mengying $\mathrm{Qi}^{1}$, Shanquan $\mathrm{Li}^{2}$, Yutong $\mathrm{Li}^{1}$, Guohua Huang ${ }^{1}$, Zuyan Fan $^{3}$, \\ Qiufeng Li $\mathbf{~}^{1}$
}

${ }^{1}$ The First Affiliated Hospital of Jinan University, Guangzhou, China

${ }^{2}$ Shenzhen Hospital of Cancer Hospital, Chinese Academy of Medical Sciences, Shenzhen, China

${ }^{3}$ The Third Affiliated Hospital of Sun Yat-Sen University, Guangzhou, China

\section{Email address:}

wuying20050511@126.com (Ying Wu),jinaihe@21cn.com (Jinai He), qimengying0605@163.com (Mengying Qi),

1sq5331@163.com (Shanquan Li), liyutong@jnu.edu.cn (Yutong Li),jndxhgh@163.com (Guohua Huang), zy.fan@163.com (Zuyan Fan), gga.2000@163.com (Qiufeng Li)

*Corresponding author

\section{To cite this article:}

Ying Wu, Jinai He, Mengying Qi, Shanquan Li, Yutong Li, Guohua Huang, Zuyan Fan, Qiufeng Li. Analysis on Related Factors and Nursing Interventions of Vasovagal Syncope During Ultrasound-guided PICC. American Journal of Nursing Science. Vol. 9, No. 4, 2020 , pp. $239-243$. doi: 10.11648/j.ajns.20200904.23

Received: June 6, 2020; Accepted: June 20, 2020; Published: June 28, 2020

\begin{abstract}
Purpose: To investigate the causes and the nursing interventions of vasovagal syncope (VVS) during ultrasound-guided Modified Seldinger Technique (MST) PICC catheterization. Methods: Clinical data (medical records, demographic data, diagnosis, local vascular compression situation, B-mode ultrasound-recorded catheter/vessel ratio and the occurrence time, symptoms and treatment of syncope) of 14 patients diagnosed as vasovagal syncope during ultrasound-guided PICC catheterization at three hospitals in Guangdong province from September 2018 to September 2019 were collected for this retrospective analysis. Results: There were 3091 cases of ultrasound-guided MST PICC catheterization in the venous catheter outpatient clinics of the 3 hospitals from September 2018 to October 2019 with 14 cases $(0.45 \%)$ of intraoperative vasovagal syncope, all cancer patients ( 5 males, 9 females) with a mean age $45 \pm 3.8$. They all received PICC for the first time and were under mental stress during the operation. Among the 14 patients ( 2 mild, 7 moderate, 5 severe), 8 had multiple lymphadenectasis in the neck and clavicular areas of the catheterization side, which resulted in different degree of venous compression and difficulty in insertion; 5 had catheter/vessel ratio over $45 \%$. 2 cases of VVS occurred when the patients were asked to turn their heads against shoulders on to the puncture side; 10 occurred when using B-mode ultrasound probe to examine the jugular vein; 2 occurred when pulling out the catheter sheath. Conclusion: The related factors of vasovagal syncope during ultrasound-guided PICC catheterization include overcompression and avulsion on blood vessels and mental stress. Therefore, it is recommended to implement psychological interventions before surgery, reduce compression and avulsion of the vascular access during catheterization, and improve practitioners' abilities in vessel selection (vessels with a catheter/vessel ratio less than $45 \%$ ) and catheterization skills to eliminate the incidence of vasovagal syncope during PICC catheterization.
\end{abstract}

Keywords: Ultrasound Guidance, Peripherally Inserted Central Catheter, Vasovaga Syncope, Cancer Patients, Cause, Nursing Interventions

\section{Background}

Central venous infusion technology has been widely applied in the treatment of severe, cancer or surgical patients [1] and peripherally inserted central catheter (PICC) has been widely used in clinical practice as well. With the help of vascular ultrasound equipment, the operation can be performed in a clear field and the status of local blood vessels can be monitored in real time. Ultrasound-guided Modified Seldinger Technique (MST) for upper arm PICC catheterization has become a routine method of PICC at home and abroad [2] which has significantly improved the success 
rate of catheterization and increase patients' satisfaction. There are many studies concerning PICC-related complications while few reports on the vasovagal syncope during ultrasound-guided PICC catheterization were found. Vasovagal syncope (VVS), a kind of nervous reflex syncope, is featured with low blood pressure, slow heart rate, dizziness, fatigue, cold sweat, pale complexion and some other symptoms. Some patients may vomit and suffer from chest tightness, temporary loss of consciousness and so on. [3] Although it has good prognosis, it increases the risks of catheterization and the psychological distress of patients. In this study, questionnaire survey was carried out among certified PICC practitioners in the venous catheter outpatient clinics of the 3 hospitals, and the clinical data of patients with VVS during PICC catheterization from September 2018 to October 2019 were collected to discuss the symptoms and causes of that and put forward possible nursing interventions.

\section{Data and Methods}

\subsection{Data Collection}

A questionnaire survey was conducted among certified
PICC practitioners in the venous catheter outpatient clinics of the 3 hospitals with 12 questionnaires $(100 \%)$ retrieved. The clinical data (medical records, demographic data, imagining materials about the status of local lymph node and vascular compression, catheter/vessel ratio, catheterization procedure and nursing records) of patients with VVS during PICC catheterization from September 2018 to October 2019 were collected and analyzed.

\subsection{Inclusion and Exclusion Criteria}

Inclusion criteria: patients diagnosed with VVS during standard ultrasound-guided MST PICC catheterization (performed with Brandentech 4Fr valve type silicone catheter and Bard 4Fr three-way valve type silicone catheter) in the 3 hospitals were included.

Exclusion criteria: patients with cardiac-cerebral-pulmonary disease, hypoglycemia, needle or blood phobia, and allergy to silicone materials.

\subsection{Grading of VVS Severity}

According to the previous study of Alboni P [4], VVS can be graded into mild, moderate and severe. (See Table 1 for details)

Table 1. Grading of VVS severity.

\begin{tabular}{ll}
\hline VVS severity & Clinical symptoms and manifestations \\
\hline Mild & Dizziness, nausea, blurred vision, general weakness, pale complexion, cold sweat \\
Moderate & A temporary loss of consciousness for less than 1 min besides mild symptoms \\
Severe & Tetany, abnormal eye movement, and loss of consciousness for more than 1 min besides mild symptoms \\
\hline
\end{tabular}

\section{Results}

There were 3091 cases of ultrasound-guided MST PICC catheterization with 14 cases $(0.45 \%)$, all cancer patients $(5$ males, 9 females) with a mean age $45 \pm 3.8$ years, all of which were under mental stress during the operation and received PICC catheterization for the first time. Among them, 8 had multiple lymphadenectasis in the neck and clavicular areas of catheterization side, which resulted in different degree of venous compression and difficulty in insertion. The vascular B-mode ultrasound showed different catheter/vessel ratio in patients $(5 \leq 45 \%, 1=45 \%, 8 \geq 45 \%)$. When the catheter was inserted 15 to $20 \mathrm{~cm}$, severe VVS occurred in 2 patients when they were required to turn their heads against shoulders on the puncture side to prevent catheter misplacement. Those 2 patients both had severe lymphadenectasis in neck and clavicular areas on the catheter side with the maximal mass of
$6.2 \times 1.5 \times 7.6 \mathrm{~cm}$ and the catheter/vessel ratio greater than $45 \%$. The operations were terminated once VVS occurred and the practitioners straightened patients' heads, applied oxygen inhalator, and slowly withdrew the catheter. The symptoms relieved after one-minute loss of consciousness. 10 patients suffered from different degree of VVS when using the ultrasound probe to examine the jugular vein after the catheter reached a predetermined length. Practitioners terminated the operation immediately, applied oxygen inhalator and pressed patients' philtrum-philtra (an acupoint to help relieve syncope or dizziness), and patients regained consciousness in less than a minute. There were totally 14 patients ( 2 mild, 7 moderate, 5 severe) with 9 patients received domestic Brandentech silicone catheters and 5 imported Bard three-way valve type catheters. All patients recovered spontaneously without any use of medication, and no positive signs remained. (See Table 2 for details)

Table 2. Clinical data of 14 patients with VVS.

\begin{tabular}{llllll}
\hline Case & Clinical diagnosis & $\begin{array}{l}\text { lymphadenectasis in } \\
\text { neck and clavicular } \\
\text { areas on the puncture } \\
\text { side }\end{array}$ & $\begin{array}{l}\text { Turning the head } \\
\text { against shoulder } \\
\text { on the puncture } \\
\text { side }\end{array}$ & $\begin{array}{l}\text { Catheter / vessel ratio } \\
\text { Occurrence time of VVS }\end{array}$ & $\begin{array}{l}\text { VVS } \\
\text { severity }\end{array}$ \\
\hline 1 & Leiomyoma of uterus & No & Yes & $31 \%$ & Pulling out catheter sheath \\
2 & Endometrial cancer & No & Yes & $30 \%$ & Pulling out catheter sheath \\
3 & Diffuse large B cell lymphoma & Yes & Yes & $30 \%$ & Pressing the neck with probe \\
4 & Nasopharynx cancer & No & Yes & $25 \%$ & Pressing the neck with probe \\
5 & Thoracic fibrosarcoma & Yes & No & $36 \%$ & Pressing the neck with probe \\
\hline
\end{tabular}




\begin{tabular}{|c|c|c|c|c|c|c|}
\hline Case & Clinical diagnosis & $\begin{array}{l}\text { lymphadenectasis in } \\
\text { neck and clavicular } \\
\text { areas on the puncture } \\
\text { side }\end{array}$ & $\begin{array}{l}\text { Turning the head } \\
\text { against shoulder } \\
\text { on the puncture } \\
\text { side }\end{array}$ & $\begin{array}{l}\text { Catheter / } \\
\text { vessel ratio }\end{array}$ & Occurrence time of VVS & $\begin{array}{l}\text { VVS } \\
\text { severity }\end{array}$ \\
\hline 6 & Squamous cell lung carcinoma & Yes & Yes & $40 \%$ & Pressing the neck with probe & Moderate \\
\hline 7 & Ovarian tumor & No & Yes & $45 \%$ & Pressing the neck with probe & Moderate \\
\hline 8 & Breast tumor & Yes & Yes & $35 \%$ & Pressing the neck with probe & Moderate \\
\hline 9 & Gastric cancer & No & Yes & $25 \%$ & Pressing the neck with probe & Moderate \\
\hline 10 & Liver cancer & Yes & No & $48 \%$ & Pressing the neck with probe & Severe \\
\hline 11 & Ovarian malignant tumor & No & Yes & $48 \%$ & Pressing the neck with probe & Severe \\
\hline 12 & Cervical cancer & Yes & Yes & $49 \%$ & Pressing the neck with probe & Severe \\
\hline 13 & T-lymphoblastoma & Yes & Yes & $68 \%$ & Turning the head against shoulder & Severe \\
\hline 14 & Breast cancer & Yes & Yes & $62 \%$ & Turning the head against shoulder & Severe \\
\hline
\end{tabular}

\section{Discussion}

\subsection{Diagnosis of VVS During Ultrasound-guided PICC}

14 patients in this group experienced VVS during ultrasound-guided MST PICC catheterization with obvious symptoms of dizziness, cold sweat, nausea, blurred vision, general weakness, pale complexion, low blood pressure and slow heart rate, and all developed temporary loss of consciousness and recovered spontaneously in about a minute without any use of medication. No positive signs remained and no history of cardio-cerebral-pulmonary disease, hypoglycemia, needle and blood phobia was found. According to the previous studies, VVS is one of the complications of ultrasound-guided visceral puncture (a minimally invasive surgery) [7], characterized by typical symptoms of dizziness, cold sweat, sudden hypotension, bradycardia and temporary loss of consciousness $[5,6]$. Most patients regain consciousness within a few seconds and possibly associated with symptoms like temporary dizziness or general weakness but without any positive signs.

\subsection{Analysis of Related Factors of VVS During PICC}

\subsubsection{Vascular Overcompression and Avulsion}

Among 14 patients with VVS, 8 had multiple lymphadenectasis in neck and clavicular areas on the puncture side. 2 of them were critical with maximal mass of $6.2 \times 1.5 \times 7.6 \mathrm{~cm}$, and both had a catheter $/$ vessel ratio greater than $45 \%$. Severe VVS occurred in these 2 patients when the catheter was inserted 15 to $20 \mathrm{~cm}$ and they were required to turn their heads against shoulders on the puncture side to prevent catheter misplacement. The INS "Infusion Therapy Standards of Practice" states that it is optimal to choose a vessel with catheter/vessel diameter ratio equal to or lower than $45 \%$ for puncture. Another 6 patients with lymphadenectasis and 4 patients without that developed VVS when using the ultrasound probe to examine their jugular vein for catheter securement after the catheter was inserted into a predetermined length, among which 3 patients with a catheter/vessel ratio greater than $45 \%$ experienced severe VVS. Therefore, we assume that the causes may be related to the lymphadenectasis on the catheter side, the catheter/vessel ratio ( $\geq 45 \%$ ), patients' position (head against shoulder), and the overcompression on blood vessels when using the ultrasound probe for examination, and the VVS will be exacerbated when these situations exist simultaneously. The vagus, widely distributed in blood vessels, has afferent and efferent pathways. The afferent sensory pressoreceptor/mechanoreceptor located in the heart and main blood vessels along with the chemoreceptor in the carotid artery collectively transmit signals to the solitary nucleus, hypothalamus and brainstem tegmental nucleus, thereby providing feedback from the cardiovascular system to the central nervous system [8]. Excessive catheter/vessel ratio mechanically stimulates the blood vessel wall; local vascular compression blocks the blood flow and increases proximal arterial tension which dilates blood vessels, thus exciting the pressoreceptor, and simultaneously causing vascular stretch reflex to stimulate vasovagal reflex. Thrasher et al. believed that afferent stimulation of vascular pressoreceptor and the stimulation of sympathetic nerve may lead to the release of adrenaline, and VVS is related to resting blood pressure that the lower the systolic pressure is, the stronger afferent activity pressoreceptor has. The duration of syncope is associated with long-time reduction of resting systolic blood pressure in supine position [9].

Overcompression and avulsion on blood vessels is one of the causes of VVS. Among the 14 patients, 2 developed mild VVS when removing the catheter sheath after the catheter was inserted into a predetermined length, which may be triggered by the mechanical stimulation of vascular endothelial cell due to overcompression and avulsion on vessels [8]. Studies showed that misoperation of catheter sheath withdrawal may cause rapid intravascular pressure drop and stimulate cerebral cortex and hypothalamus which dilates the small vessels and eventually results in VVS when patients develop low blood pressure and slow heart rate.

\subsubsection{Mental Stress}

Mental stress is also one of the causes of VVS. Studies showed that mental stress is a main cause of vagal reflex in patients undergoing vascular intervention [10]. PICC catheterization is an invasive operation on blood vessels and many patients suffer from mental stress because they have limited knowledge about the operation. Patients' physical functions may be compromised if they are in a state of tension, anxiety and fear [11] that may stimulate the vascular 
endothelial system and a greater stretch on vasovagal nerve and eventually trigger vagal reflex. All 14 patients in this group were under mental stress before surgery for first time catheterization which stimulated the release of prostaglandin or bradykinin thus exciting vagus nerve. Ritsuko believed that early significant increase of plasma adrenaline levels from the baseline is associated with short-time VVS, and trials suggested that it may be related to the susceptibility of VVS [12].

\subsection{Nursing Interventions}

\subsubsection{Preoperative Examination}

It is recommended to examine vessels by ultrasound equipment and select vessels with a catheter/vessel diameter ratio of $45 \%$ or less [13]. Imaging examinations should be done as well to see whether there is lymphadenectasis in the neck and clavicular areas to avoid puncture in the pressured arm to reduce complications.

\subsubsection{Psychological Intervention Before Catheterization}

Psychological assessment should be carried out on patients and their families before catheterization. Due to the lack of relevant medical knowledge, patients are prone to feel stressful and fear for invasive procedures, which increases the incidence of VVS. Therefore, preoperative psychological counseling becomes essential to explain the purpose, significance and process of ultrasound-guided PICC to patients and their families for better cooperation. Music therapy can be used to relieve patients' anxiety.

\subsubsection{Avoid Overcompression and Avulsion on Blood Vessels}

Practitioners should use B-mode ultrasound probe in a proper strength for examination to avoid overcompression on the blood vessels, and remove catheter sheath slowly and gently then press the blood vessels to stop bleeding, avoiding avulsion on blood vessels due to quick and vigorous withdrawal.

\subsubsection{Treatment of Vasovagal Syncope During Catheterization}

Patients' condition shall be constantly monitored during catheterization. The catheter should be withdrawn once symptoms like dizziness, nausea, blurred vision, tinnitus, hearing loss, pale complexion and cold sweat occurred, and patient's head shall be straightened to eliminate any possible triggers of VVS. When the patients lose consciousness, oxygen inhalator and vital signs monitoring should be applied immediately, and the patients should be kept in Trendelenburg position to increase cerebral blood supply to ensure their safety and the doctors should be notified at the same time. Practitioners can also press philtrum-philtra, Hegu and Neiguan points to help patients regain consciousness and shorten the duration of syncope. Most patients can regain their consciousness spontaneously within minutes. After that, patients' vital signs should be reassessed, and they should rest on bed for no less than 30 minutes to prevent relapse. For those who fail to regain consciousness within $15 \mathrm{~s}$, emergency treatments should be taken immediately following doctors' advice, and the rescue process should be recorded.

\subsubsection{Assessment of Catheterization Practitioners}

The comprehensive abilities of catheterization practitioners should be assessed, such as the ability for overall assessment of patients, especially the assessment of VVS-related factors. Training should be carried out to improve their puncture skills and proficiency, and to improve the their abilities to detect and deal with the complications during PICC, thereby reducing the risks of catheterization.

\section{Conclusion}

Vasovagal syncope (VVS) is one of the acute and serious complications during ultrasound-guided PICC. Although it has low incidence and most patients can recover spontaneously, it progresses rapidly once occurs and increases the risks of catheterization and mental stress for patients. This study suggests that the related factors of vasovagal syncope during ultrasound-guided PICC catheterization include overcompression and avulsion of blood vessels on the catheter side, and mental stress. Active interventions to eliminate the incidence of VVS include taking preoperative psychological interventions, avoiding catheterization in the neck and clavicular areas with lymphadenectasis, avoiding overcompression and avulsion on blood vessels when using the ultrasound probe and withdrawing catheter sheath, paying close attention to patients' conditions during operation. Besides, the risks of PICC catheterization can be reduced through active nursing interventions. In conclusion, the practitioners should terminate the operation once VVS occurs, withdraw the catheter and perform first aid immediately. Further researches are required due to the limited samples in this study.

\section{Acknowledgements}

We thank for Jinai He for assistance Analysis on Related Factors and Nursing Interventions of Vasovagal Syncope during Ultrasound-guided PICC

\section{References}

[1] Johansson E, Hammarskjöld F, Lundberg D, et al. Advantages and disadvantages of inserted central venous catheters (PICC) compared to other central venous lines: a systematic review of the literature [J]. Acta oncologica, 2013, 52 (5): 886-892.

[2] Hunter M. Peripherally inserted central catheter placement: the speed of sound [J]. Nutrition in Clinical Practice, 2007, 22 (4): 406-411.

[3] SLOAN H E. The vagus nerve in cardiac arrest; the effect of hypercapnia, hypoxia and asphyxia on reflex inhibition of the heart [J]. Surgery, gynecology \& obstetrics, 1950, 91 (3): 257-264.

[4] Alboni P. The different clinical presentations of vasovagal syncope. Heart, 2015, 101 (9): 674-8. 
[5] Brignole M, Moya A, de Lange FJ, Deharo JC, Elliott PM, Fanciulli A, Fedorowski A, FurlanR, Kenny RA, Martin A, Probst V, Reed MJ, Rice CP, Sutton R, Ungar A, van Dijk JG; ESC Scientific Document Group. 2018 ESC Guidelines for the diagnosis and management of syncope. Eur Heart J. 2018; 39 (21): 1883-1948.

[6] Shen WK, Sheldon RS, Benditt DG, Cohen MI, Forman DE, Goldberger ZD, Grubb BP, Hamdan MH, Krahn AD, Link MS, Olshansky B, Raj SR, Sandhu RK, Sorajja D, Sun BC, Yancy CW. 2017 ACC/AHA/HRS Guideline for the Evaluation and Management of Patients With Syncope: Executive Summary: A report of the American College of Cardiology/American Heart Association Task Force on Clinical Practice Guidelines and the Heart Rhythm Society. Circulation. 2017; 136 (5): e25-e59.

[7] Humm AM, Z'Graggen WJ. Venepuncture during head-up tilt testing in patients with suspected vasovagal syncope implications for the test protocol [J]. Eur J Neurol, 2015; 22 (2): 389-94.

[8] Olshansky B. Vagus nerve modulation of inflflammation: Cardiovascular implications [J]. Trends Cardiovasc Med, 2016, 26 (1): 1-11.
[9] Thrasher TN. Baroreceptors, baroreceptor unloading, and the long-term control of blood pressure [J]. Am J Physiol Regul Integr Comp Physiol. 2005; 288 (4): R819- R827.

[10] Huston J M, Fritz J R. The inflammatory reflex and neural tourniquet: harnessing the healing power of the vagus nerve $[\mathrm{J}]$. 2018, 1 (1): 29-38.

[11] Inoue T, Abe C, Sung SS, Moscalu S, Jankowski J, Huang L, Ye H, Rosin DL, Guyenet PG, Okusa MD. Vagus nerve stimulation mediates protection from kidney ischemia-reperfusion injury through $\alpha 7 \mathrm{nAChR}+$ splenocytes [J]. J Clin Invest, 2016, 126 (5): 1939-52.

[12] Kohno R, Detloff BLS, Chen LY, Norby FL, Benditt DG. Greater early epinephrine rise with head-up posture: A marker of increased syncope susceptibility in vasovagal fainters [J]. J Cardiovasc Electrophysiol, 2019, 30 (3): 289-296.

[13] The 2016 Infusion Therapy Standards of Practice [J]. Home Healthcare Now, 2017, 35 (1): E1-E2. DOI: 10.1097/NHH.0000000000000504. 